\title{
Preliminary Evaluation of Atmospheric Temperature and Wind Profiles Obtained Using Unmanned Aerial Vehicle Based Acoustic Tomography
}

\author{
Anthony Finn*, Kevin Rogers, Joshua Meade, Jarrod Skinner, Amir Zargarian
}

School of Engineering, University of South Australia, Mawson Lakes, Australia, SA 5095 - (\{kevin.rogers\}, \{joshua.meade $\}$, \{jarrod.skinner\}, \{amirhossein.zargarian\}@ mymail.unisa.edu.au)

Commission I, WG I/II

KEY WORDS: UAV, Remote Sensing, Tomography, LIDAR, Atmospheric Boundary Layer, Acoustic Propagation

\begin{abstract}
:
An acoustic signature generated by an unmanned aerial vehicle is used in conjunction with tomography to remotely sense temperature and wind profiles within a volume of atmosphere up to an altitude of $120 \mathrm{~m}$ and over an area of $300 \mathrm{~m} \times 300 \mathrm{~m}$. Sound fields recorded onboard the aircraft and by an array of microphones on the ground are compared and converted to sound speed estimates for the ray paths intersecting the intervening medium. Tomographic inversion is then used to transform these sound speed values into threedimensional profiles of virtual temperature and wind velocity, which enables the atmosphere to be visualised and monitored over time. The wind and temperature estimates obtained using this method are compared to independent measurements taken by a co-located mid-range ZephIR LIDAR and sensors onboard the aircraft. These comparisons show correspondences to better than $0.5^{\circ} \mathrm{C}$ and $0.3 \mathrm{~m} / \mathrm{s}$ for temperature and wind velocity, respectively.
\end{abstract}

\section{INTRODUCTION}

Good temperature and wind-speed measurements are needed to identify and examine the properties of the atmospheric boundary layer (ABL). Features of interest include convective structures in a daytime ABL, the nocturnal low-level jet, elevated inversions and temperature structures, turbulence within the ABL, sea breeze circulations, gravity waves, frontal passages, and wind flow over complex terrain. Several methods for wind energy observation exist: cup and sonic anemometers (usually mounted on masts), sound detection and ranging (SODAR), light detection and ranging (LIDAR), radio acoustics sounding system (RASS), radar, satellite-based techniques and radiosondes. Acoustic atmospheric tomography (AAT) has also been used to observe virtual temperature and wind velocity profiles of the atmosphere, and to monitor their evolution in time and space. Each has benefits and drawbacks and an overview of AAT is available in (Ostashev et al., 2008, Ostashev and Wilson, 2016).

AAT has historically been restricted to near-horizontal atmospheric soundings close to ground level $(<50 \mathrm{~m})$. Based on observations made during the overflight of a UAV, techniques have been developed that allow determination of temperature and wind profiles up to altitudes of about $2 \mathrm{~km}$ (Finn and Rogers, 2015, Finn and Rogers, 2016b); and techniques for acoustically tracking certain UAVs at ranges in excess of $4 \mathrm{~km}$ have recently been demonstrated (Finn et al, 2019). This suggests the ability to monitor significant volumes of atmosphere, although geometric and tomographic considerations will constrain accuracies.

The approach potentially overcomes limitations such as short operating ranges suffered by mid-range SODAR and LIDAR instruments (typically $<300 \mathrm{~m}$ for medium performance systems); and it negates the need to horizontally interpolate or extrapolate wind speed and variance from such observations.
Multi-UAV and air-underwater techniques are also available (Finn and Rogers, 2016a, Finn and Rogers, 2017). Moreover, if 2D microphone arrays are used, 3D tomographic profiles may be reconstructed (Rogers and Finn, 2013); and, as equipment costs a fraction of a LIDAR, SODAR or mast, deployment in inaccessible or hazardous locations is more justifiable. There are, of course disadvantages to UAV-based AAT, such as the current legislated need for manned operation (for safety reasons), that precludes extended (months/years) campaign deployments.

Contribution: The performance of 2D UAV-based AAT has previously been examined using synthetic atmospheres generated using radial basis functions, large eddy simulation and SODAR (Finn et al 2017). UAV-based AAT estimates of wind and temperature have never been assessed against LIDAR, in campaigns where wind speeds have exceeded $5 \mathrm{~m} / \mathrm{s}$, or where temperature measurements were obtained concurrently; and 3D UAV-based AAT estimates have never been assessed against independent instruments. This paper reports on all these.

\section{COMPARISONS BETWEEN UAV-BASED AAT AND LIDAR}

SODAR, LIDAR and radar operate using similar principles: SODAR emit acoustic energy, LIDAR near infrared light energy and radar radio energy. The scatterers are turbulent refractivity fluctuations, particulate matter carried by the atmosphere and turbulent eddies whose scale size are half the wavelength of the emitted signal, respectively. All then obtain wind velocity and height information from the Doppler shift and time delay information derived from the signal scattered back to the receiver. Similar assumptions are made regarding homogeneity of scatterers and the manner in which they are carried by the wind. For a more complete understanding of the operating principles of these instruments the reader is referred to (Hall Jr et

\footnotetext{
* Corresponding author (anthony.finn@ @unisa.edu.au)
} 
al., 1984, Hooper and Eloranta, 1986, Singal, 1997, Antoniou et al., 2003, May et al., 1989, May et al., 1988, May et al., 1990, Strauch et al., 1984).

UAV-Based AAT operates by recording sound fields onboard the aircraft and at an array of microphones on the ground. These are compared and time delays for each intersecting ray path penetrating the intervening atmosphere computed and converted to sound speed measurements. The relationship between temperature, wind velocity and sound speed is then exploited and tomographic inversion used to transform these sound speed values into three-dimensional profiles of virtual temperature and wind velocity.

Tomography is a sub-set of inverse theory from which a data kernel is formed by integrating the model parameters, $\mathrm{m}$, along the ray paths that intersect a medium. Measurements, b_obs, of observed the time delays are used to infer values of the sound speed (model) parameters. The model and the data set are related by a set of explicit equations, b_obs=a(m), which may be written as, b_obs=Am, if the relationship between model parameters is linear, where A is the data kernel. The model used to compute the tomographic inversions in this paper is described in detail in (Finn and Rogers, 2015, Finn and Rogers, 2016b, Rogers and Finn, 2016).

The resultant profiles are represented by radial basis functions (Wiens, 2008). This enables the atmosphere to be visualised in high resolution and monitored over time in 3D. In order for the technique to work, estimates of propagation delay must be accurate to within $\pm 0.1 \mathrm{~ms}$ and UAV positions known to within $\pm 5 \mathrm{~cm}$ for any epoch (Rogers and Finn, 2017).

Over a period of 5 days, field trials were conducted near Rowland Flat in South Australia. The days were hot $\left(\max >40^{\circ} \mathrm{C}\right)$ with moderate winds $(5-10 \mathrm{~m} / \mathrm{s})$. A DJI Matrice 600 UAV was repeatedly flown at an altitude of up to $120 \mathrm{~m}$ above a $300 \mathrm{~m} \mathrm{x}$ $200 \mathrm{~m}$ array of 35 microphones. The inter-sensor separation distances for the microphones was approximately $50 \mathrm{~m}$. All were positioned approximately $1.5 \mathrm{~m}$ above ground level. The ground was flat with variation in elevation of about $12 \mathrm{~m}$. The vegetation was vines in full leaf (close to harvest).

The location of each microphone was determined using Real Time Kinematic Carrier Phase Differential Global Positioning System, which has an accuracy of $\pm 0.03 \mathrm{~m}$. The UAV was also fitted with RTK CP DGPS, enabling position recording at $5 \mathrm{~Hz}$ with similar accuracy. Horizontal wind velocity, air temperature, barometric pressure and relative humidity were also recorded onboard the UAV at $1 \mathrm{~Hz}$.

The ground array comprised ECM $80010 \mathrm{mV} / \mathrm{Pa}$ condenser microphones sampled at $44.1 \mathrm{kHz}$ using four 8 -channel 24-bit Data Acquisition (DAQ) recorders with $107 \mathrm{~dB}$ spurious free dynamic range. The DAQ sampling frequencies drift with temperature and cannot be relied upon to provide accurate timestamping. Hence, one of the DAQ channels recorded a GPSderived 1PPS signal to provide accurate absolute timing. A similar 2-channel DAQ/microphone setup was carried onboard the UAV.

A ZephIR LIDAR was located outside the microphone array, approximately $50 \mathrm{~m}$ northwest of the sensor coinciding with the origin of the coordinate system. 3D wind velocities were observed at $10 \mathrm{~m}$ intervals between altitudes of $30 \mathrm{~m}$ and $110 \mathrm{~m}$ every $15 \mathrm{sec}$. The $(1 \sigma)$ nominal uncertainty for measurement error reported by the manufacturer is $0.3 \mathrm{~m} / \mathrm{s}$ for each axis, but this figure is generally dependent upon the prevailing conditions and particulate matter contained by the atmosphere. A more detailed assessment was not made.

A DJI Phantom IV UAV carrying two iMet-XQ sensors was repeatedly flown around the perimeter of the microphone array between an altitude of $20 \mathrm{~m}$ and $120 \mathrm{~m}$. Two sensors were used as there is typically up to $0.5^{\circ} \mathrm{C}$ variation between the two sensors, depending upon their placement on the UAV (Jacob et al., 2018).

Position based on SPS GPS, thermodynamic temperature, pressure, and relative humidity were recorded at $1 \mathrm{~Hz}$. Pressure was used (in conjunction with a third, ground-based iMet-XQ) to determine sensor altitude to within $\pm 0.5 \mathrm{~m}$ as the vertical component of SPS GPS is generally accurate only to about $\pm 20 \mathrm{~m}$. Thermodynamic temperature was converted to virtual temperature based on measurements taken onboard the UAV. The accuracy of the temperature sensor is $\pm 0.3^{\circ} \mathrm{C}$.

3D temporally averaged volumetric atmospheric profiles for wind velocity and temperature were obtained using UAV-based AAT. These are compared to the independent measurements taken by the LIDAR and sensors onboard the Phantom UAV (Figure 1, Figure 2, and Figure 3).

In Figure 1 and Figure 2 the vertical red line represents the temporal average of the LIDAR data over the duration of the entire AAT observation set, whereas the green dots represent the AAT estimates at each $10 \mathrm{~m}$ increment for the location closest to the LIDAR. The (red) error bars associated with the LIDAR data represent $\mathrm{min} / \mathrm{max}$ variation of the LIDAR measurements over the observation period of the AAT, whereas the blue bars represent the min/max variation of the AAT estimates over the area of the array at each $10 \mathrm{~m}$ interval.

In Figure 3 the differences between the mean of the virtual temperatures observed by the two iMet sensors onboard the Phantom UAV and the AAT estimates are displayed. They are colour-coded in accordance with the scale on the right of each image. The coordinate system is such that the origin coincides with the leftmost microphone of the array. The positive y-axis points forward through the left-most line of microphones, the $\mathrm{z}$ axis is vertical and the $\mathrm{x}$-axis orthogonal to these two axes, roughly coinciding with the first microphone in each row.

\section{SUMMARY}

Three-dimensional, temporally averaged volumes of atmosphere are obtained by: monitoring sounds generated by a UAV as it overflies an array of ground microphones; comparing the received signal to that predicted using onboard measurements; generating time delay observations for the multiple intersecting ray paths that penetrate the intervening atmosphere; and converting the computed sound speed measurements to temperature and wind fields represented by radial basis functions using tomographic inversion. These profiles are compared to independent measurements taken by another UAV and by a ZephIR LIDAR.

The AAT and UAV/LIDAR measurements show correspondence accuracies of around $0.5^{\circ} \mathrm{C}$ for temperature and $0.3 \mathrm{~m} / \mathrm{s}$ for each component of wind velocity, respectively. This compares very favourably to other inter-instrument atmospheric comparisons, such as LIDAR vs. SODAR and is within the measurement errors of the system. Nevertheless, it is difficult to conclusively establish performance envelope for UAV-based AAT because: 
- The test data set is sparse, comprising data over a period of only a few days, with wind speeds extending up to only $10 \mathrm{~m} / \mathrm{s}$.

- LIDAR and AAT observe fundamentally different atmospheric properties: LIDAR observes the time delay from a signal emitted and backscattered by particulate matter that is assumed to travel with the wind, whereas AAT relates a one-way propagation delay of a signal to sound speed;

- The integration period of the LIDAR used in this experiment differs substantially from the AAT observation period. Thus, both provide vector sums of wind velocity over their respective (and very different) observation periods;
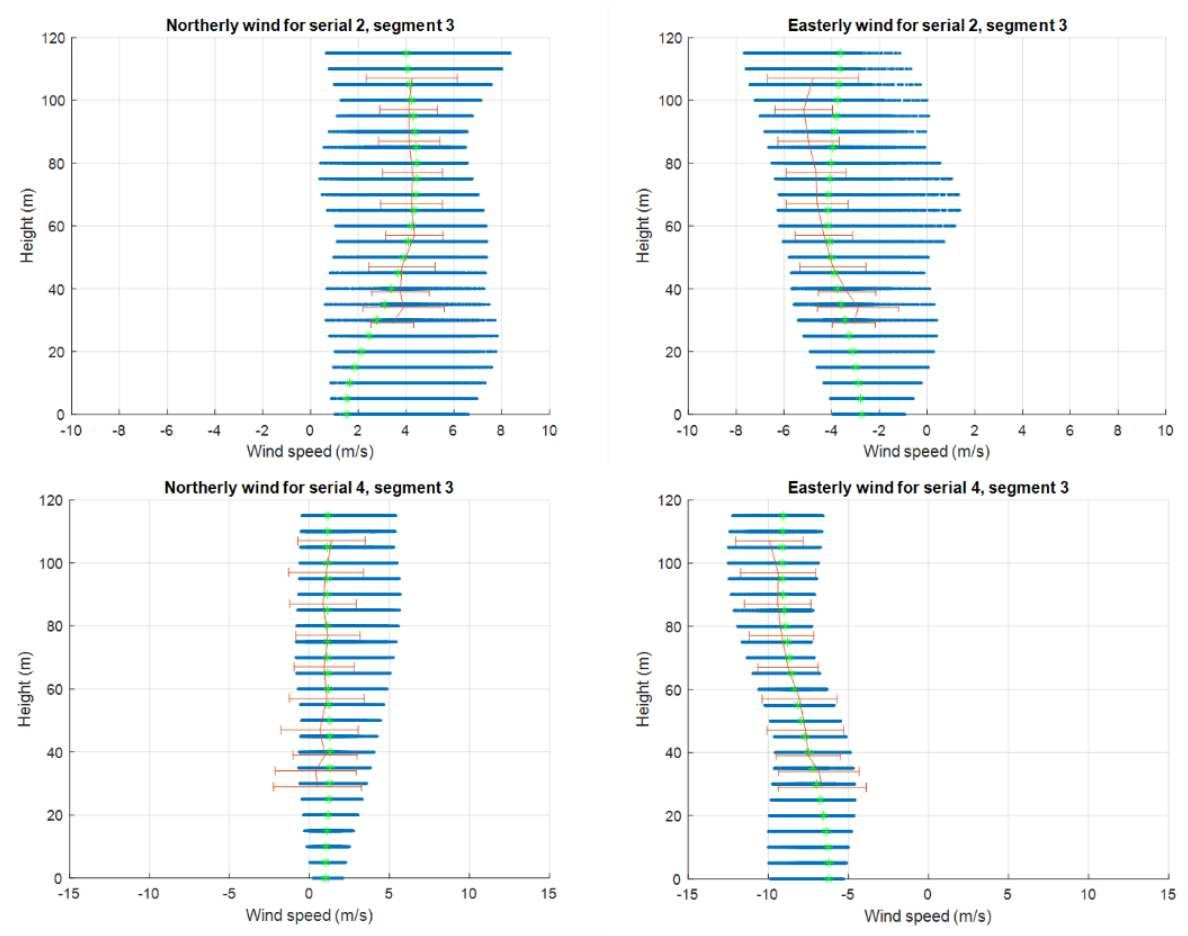

Figure 1: Comparison between horizontal wind speed components observed by LIDAR (red) and UAV-based AAT (green)
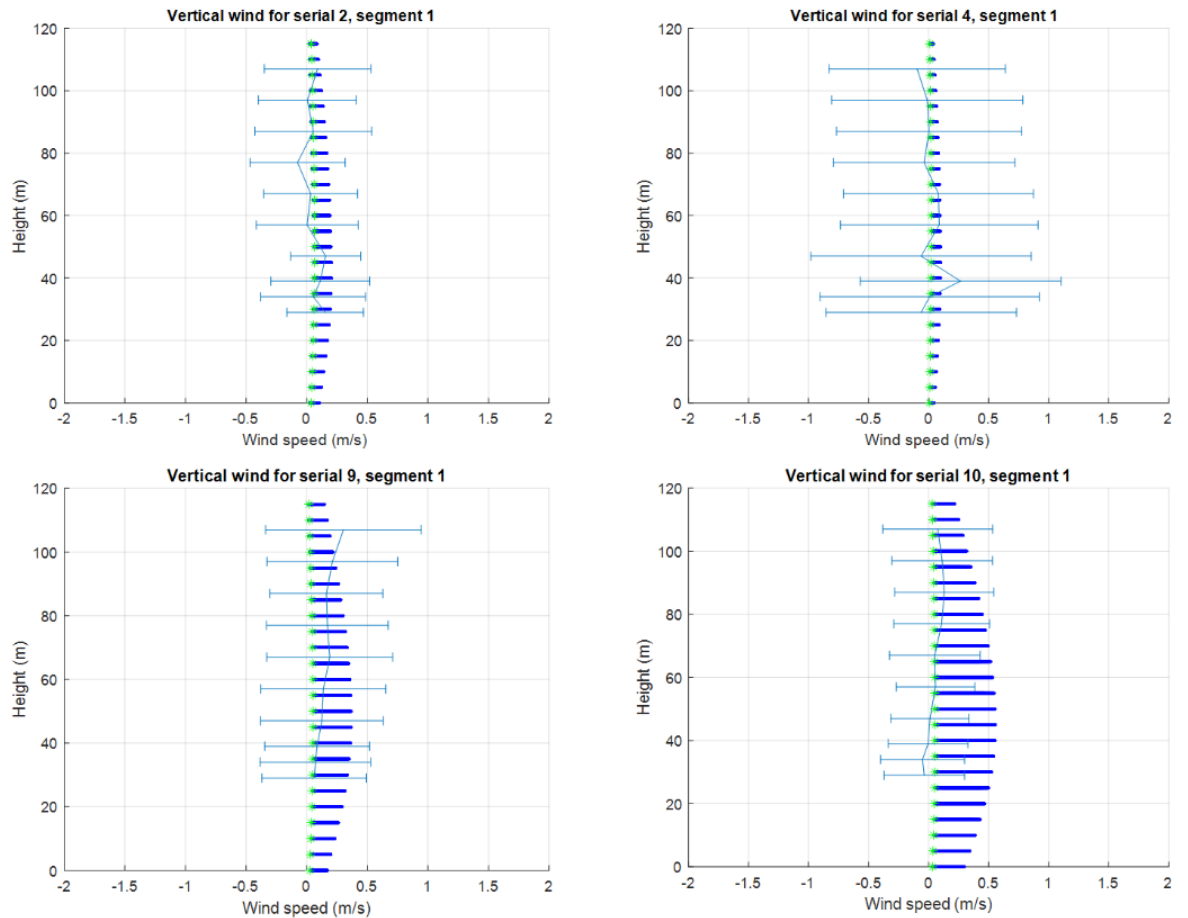

Figure 2: Comparison between vertical wind speed components observed by LIDAR (light blue) and UAV-based AAT (green)
UAV-based AAT observations are affected by the effects of refraction, which is known to be imperfectly modelled;

AAT permits visualisation of atmospheric profiles over $600 \mathrm{~m}$ baselines and up to $1,200 \mathrm{~m}$, although the accuracy of the horizontal wind velocity estimates appear to diminish when the altitude of the UAV overflight is greater than $600 \mathrm{~m}$, although vertical wind velocity estimates appear to improve slightly for these higher flights. 

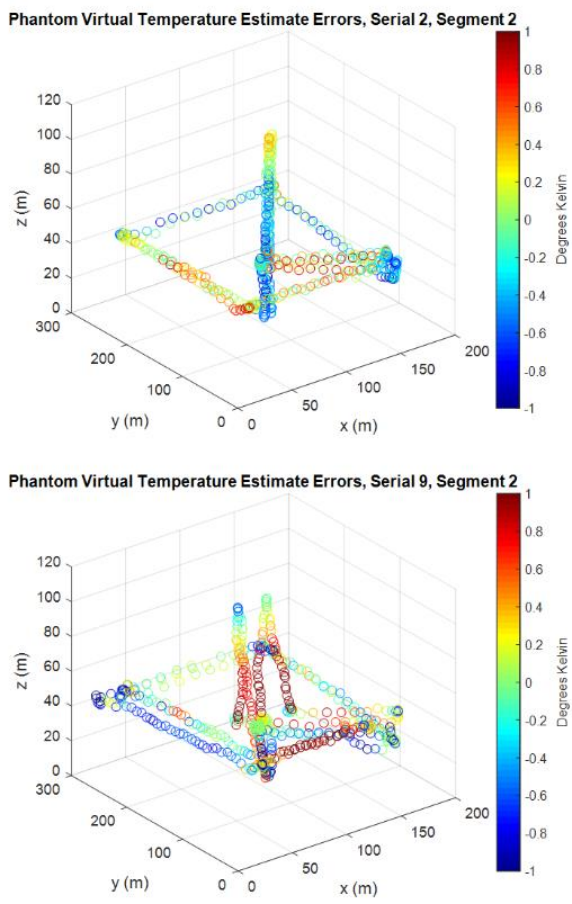
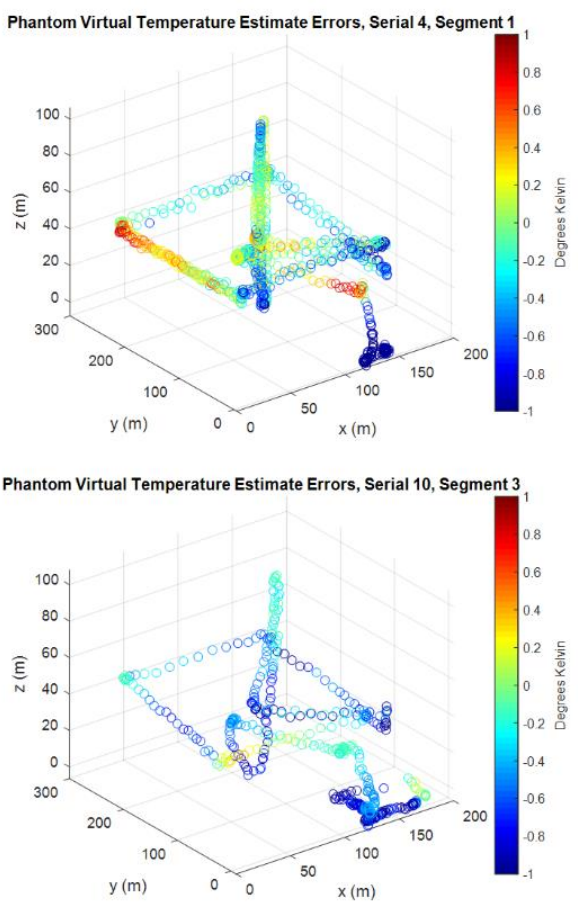

Figure 3: Comparison between UAV-based AAT temperature estimates and those observed independently by another UAV

\section{ACKNOWLEDGEMENTS}

Funding: This research was funded by Wine Australia, grant number USA1601, "Use of Unmanned Air Vehicles for early, real time detection of extreme weather events in vineyards"

Acknowledgments: We are grateful to Treasury Wine Estates, Pernod Ricard and Barton Vale Technologies - in particular Dr Catherine Kidman, Tim McCarthy and Peter Smith — for their collaboration and Steve Andriolo of EyeSky for UAV operations and trials support.

\section{REFERENCES}

Antoniou, I., Jørgensen, H. E., Ormel, F., Stuart Bradley, Hünerbein, S. V., Emeis, S. \& Warmbier, G. (2003) On the Theory of SODAR Measurement Techniques. Roskilde, Denmark.

Finn, A. \& Rogers, K. (2015) The feasibility of unmanned aerial vehicle-based acoustic atmospheric tomography. J Acoust Soc Am 138(2):874-889.

Finn, A. \& Rogers, K. (2016a) Acoustic Atmospheric Tomography Using Multiple Unmanned Aerial Vehicles. IET Radar, Sonar \& Navigation 10:1541-1551.

Finn, A. \& Rogers, K. (2016b) Improving Unmanned Aerial Vehicle-Based Acoustic Atmospheric Tomography by Varying the Engine Firing Sequence of the Aircraft. Journal of Atmospheric and Oceanic Technology 33(4):803-816.

Finn, A. \& Rogers, K. (2017) An Acoustic Tomography Technique for Concurrently Observing the Structure of the Atmosphere and Water Bodies. Journal Atmospheric \& Oceanic Technology 34(3):617-629.
Finn, A., Rogers, K., Rice, F., Meade, J., Holland, G. \& May, P. (2017) A Comparison of Vertical Atmospheric Wind Profiles Obtained from Monostatic SODAR and Unmanned Aerial Vehicle Based Acoustic Tomography. Journal Atmospheric \& Oceanic Technology 4(10):18.

Finn, A., R. Wyber, J. Fang \& R. Brinkworth, Acoustic Detection of UAV from Ground Microphones, Report to Defence Science \& Technology Group, DASI Technical Note 1-2019, 2019

Hall Jr, F. F., Huffaker, R. M., Hardesty, R. M., Jackson, M., Lawrence, T. R., Post, M. J., Richter, R. \& Weber, B. (1984) Wind measurement accuracy of the NOAA pulsed infrared Doppler lidar. Applied optics 23(15):2503-2506.

Hooper, W. P. \& Eloranta, E. W. (1986) Lidar measurements of wind in the planetary boundary layer: the method, accuracy and results from joint measurements with radiosonde and kytoon. Journal of climate and applied meteorology 25(7):990-1001.

Jacob, J. D., Chilson, P. B., Houston, A. L. \& Smith, S. W. (2018) Considerations for Atmospheric Measurements with Small Unmanned Aircraft Systems. Atmosphere 9(7).

May, P. T., Moran, K. P. \& Strauch, R. G. (1989) The accuracy of RASS temperature measurements. J. Appl. Meteor. 28:13291335 .

May, P. T., Strauch, R. G. \& Moran, K. P. (1988) The altitude coverage of temperature measurements using RASS with wind profiler radars. Geophys. Res. Lett. 15:1381-1384.

May, P. T., Strauch, R. G., Moran, K. P. \& Ecklund, W. L. (1990) Temperature sounding by RASS with wind profiler radars: A preliminary study. IEEE Trans. Geosci. Remote Sens 28:19-28.

Ostashev, V., Vecherin, S. N., Wilson, D. K., Ziemann, A. \& Goedecke, G. H. (2008) Recent progress in acoustic tomography 
of the atmosphere In Proceedings of IOP Conf. Series: Earth Env. Sci. IOP Publishing vol. 1, pp. 012008.

Ostashev, V. \& Wilson, K. D. (2016) Acoustics in moving inhomogeneous media (second edition). CRC Press, Taylor \& Francis.

Rogers, K. \& Finn, A. (2013) Three-Dimensional UAV-Based Atmospheric Tomography. J. Atmos. \& Oceanic Techn. 30(2):336-344.

Rogers, K. \& Finn, A. (2016) Accurate sound speed estimation for Unmanned Aerial Vehicle-Based Acoustic Atmospheric Tomography In Proceedings of Proceedings of ACOUSTICS 2016. Australaian Acoustical Society.

Rogers, K. \& Finn, A. (2017) Accurate sound speed estimation for unmanned aerial vehicle based acoustic atmospheric tomography. J Acoust Soc Am 141(2):1269-1280.

Singal, S. P. (1997) Acoustic Remote Sensing Applications. Heidelberg, Springer-Verlag.

Strauch, R. G., Merritt, D. A., Moran, K. P., Earnshaw, K. B. \& Kamp, D. V. D. (1984) The Colorado Wind Profiling Network. Journal Atmospheric \& Oceanic Technology 1:37-49.

Wiens, T. (2008) Sensing of turbulent flows using real-time acoustic tomography In Proceedings of 19th Biennial Conf. of the New Zealand Acoustical Society.

Revised February 2019 